POS $\quad$ PROCEEDINGS

\title{
Holographic superconductors at finite isospin density or in an external magnetic field
}

\author{
Johanna Erdmenger*, Patrick Kerner and Migael Strydom \\ Max-Planck-Institut für Physik (Werner-Heisenberg-Institut), \\ Föhringer Ring 6, 80805 München, Germany \\ E-mail: jke, pkerner, mstrydom @mppmu.mpg.de
}

\begin{abstract}
We review recent work on realizing a superfluid within gauge/gravity duality in the top-down approach, based on embedding a probe of two coincident D7 branes into the AdS-Schwarzschild black hole geometry. From the QCD point of view, this corresponds to a flavour superfluid in the presence of a finite isospin density. Moreover, inspired by recent results within QCD on superconductivity in presence of an external magnetic field, we also investigate the possibility of a magnetic field triggering superfluidity within a similar holographic approach.
\end{abstract}

The many faces of $Q C D$

November 2-5, 2010

Gent Belgium

${ }^{*}$ Speaker. 


\section{Introduction}

In 2008, a simple model for a holographic superconductor was proposed within gauge/gravity duality, based on the condensation of a charged scalar in a charged Anti-de Sitter black hole geometry [1,2]. In this model, the frequency-dependent conductivity displays the features expected for a superconductor. Numerous generalisations of this model have been studied, in particular a p-wave superconductor in which a component of an SU(2) gauge field condenses [3].

The p-wave superconductor model may be realised in a ten-dimensional low-energy string theory construction by embedding a probe of two coincident D7 branes into the AdS-Schwarzschild background $[4,5]$. A finite isospin chemical potential and density are turned on by considering a non-trivial profile for the $\mathrm{SU}(2)$ gauge field on the brane probe. This construction has the advantage that the dual four-dimensional field theory is explicitly known; it is given by $\mathscr{N}=4$ Super-YangMills theory plus two $\mathscr{N}=2$ fundamental hypermultiplets, the supersymmetry being broken by the finite temperature and the finite isospin density. The condensate obtained in this theory is a charged $\rho$ meson condensate. String theory embeddings of the scalar or s-wave superconductor have been discussed for instance in $[6,7]$. A $\rho$ meson condensate for the Sakai-Sugimoto holographic model has been found in [8].

In our model of $[4,5]$, we calculated the frequency-dependent conductivity and showed that it has the behaviour expected for a superconductor. We confirmed that the new condensate state has lower free energy. We also found a remnant of the Meissner-Ochsenfeld effect in an external magnetic field: since the spontaneously broken symmetry is global, the Meissner effect just implies that the superfluid condensate vanishes above a critical value for the magnetic field, which depends on the temperature. For a related probe D-brane model, fermionic excitations were studied in [9]. In the superfluid phase, the Fermi surface splits into isolated peaks in momentum space. These correspond to the zeros of the condensate on the normal state Fermi surface.

At the workshop 'The many faces of QCD' we were given the opportunity to discuss our results with M. Chernodub and to compare them to his recent results within QCD [10, 11], see also [12], in which he found a superconducting state within QCD in a strong magnetic field. These discussions motivated us to look at a similar mechanism in the holographic setup with D7 brane probes as described above. In fact, introducing an external magnetic field is technically very similar to introducing an isospin chemical potential: the magnetic field requires introducing a non-trivial profile for the spatial component $A_{y}^{3}$ of the gauge field on the brane, with the upper index denoting the $\mathrm{SU}(2)$ component, while the isospin chemical potential requires a temporal gauge field profile $A_{t}^{3}$. Below, we argue that a superfluid ground state may also be found by using the background corresponding a magnetic field in the holographic approach.

\section{Holographic superfluid at finite isospin density}

Let us first discuss the finite isospin case. We start with a ten-dimensional geometry involving an 4+1 dimensional AdS-Schwarzschild black hole as well as a five-sphere, corresponding to $\mathscr{N}=$ 4 Super Yang-Mills theory a finite temperature. The metric is given by

$$
d s^{2}=\frac{r^{2}}{2 R^{2}}\left(-\frac{f^{2}}{\tilde{f}} d t^{2}+\tilde{f} d \vec{x}^{2}\right)+\left(\frac{R}{r}\right)^{2}\left(d r^{2}+r^{2} d \Omega_{5}^{2}\right),
$$


with

$$
f(r)=1-\frac{r_{H}^{4}}{r^{4}} \quad \tilde{f}(r)=1+\frac{r_{H}^{4}}{r^{4}} .
$$

$R$ is the AdS radius given by $R^{4}=4 \pi g_{S} N_{c} \alpha^{\prime 2}=2 \lambda \alpha^{\prime 2}$ and $r_{H}$ is the radius at which the event horizon is found. The Hawking temperature of the system is given by $T=\frac{r_{H}}{\pi R^{2}}$, which is also the temperature of the field theory on the boundary.

To include fundamental matter, we embed $N_{f}$ coincident D7-branes into the ten-dimensional spacetime. For simplicity, we consider massless embeddings (corresponding to massless quarks) only. In this case the D7-brane wraps $A d S_{5} \times S^{3}$. These D7-branes host flavor gauge fields $A_{\mu}$ with gauge group $U\left(N_{f}\right)$. We introduce an $S U(2)$ isospin chemical potential $\mu$ by a non-vanishing time component of the non-Abelian background field on the D7-brane. The generators of the $S U(2)$ gauge group are given by the Pauli matrices $\sigma^{i}$. Due to the gauge symmetry, we may rotate the flavor coordinates until the chemical potential lies in the third flavor direction,

$$
\mu=\lim _{r \rightarrow \infty} A_{t}^{3}(r) .
$$

This non-zero gauge field breaks the $S U(2)$ gauge symmetry down to $U(1)_{3}$ generated by the third Pauli matrix $\sigma^{3}$. The spacetime symmetry on the boundary is still $S O(3)$ since the Lorentz group $S O(3,1)$ is already broken down to $S O(3)$ by the finite temperature. The chemical potential $(2.3)$ is the source for the operator

$$
J_{t}^{3} \propto \bar{\psi} \sigma^{3} \gamma_{t} \psi+\phi \sigma^{3} \partial_{t} \phi=n_{u}-n_{d},
$$

where $n_{u / d}$ is the charge density of the isospin fields.

In addition, we consider a further non-vanishing background gauge field which stabilises the system for large chemical potentials. Due to the symmetry of our setup we may choose $A_{y}^{1} \mathrm{~d} y \sigma^{1}$ to be non-zero. To obtain an isotropic configuration in the field theory, this new gauge field $A_{y}^{1}$ only depends on $r$. Due to this two non-vanishing gauge fields, the field strength tensor on the branes has the non-zero components,

$$
\begin{aligned}
F_{r y}^{1} & =-F_{y r}^{1}=\partial_{r} A_{y}^{1}, \\
F_{t y}^{2} & =-F_{y t}^{2}=\frac{\gamma}{\sqrt{\lambda}} A_{t}^{3} A_{y}^{1}, \\
F_{r t}^{3} & =-F_{t r}^{3}=\partial_{r} A_{t}^{3} .
\end{aligned}
$$

The equations of motion which determine the profile of the D7-brane probes and of the gauge fields on these branes are obtained from the Dirac-Born-Infeld (DBI) action. Since we require two coincident D7 branes for the necessary SU(2) symmetry to ensure a charged condensate, the DBI action is necessarily non-abelian. By using the spatial and gauge symmetries present in our setup, we obtain a non-abelian DBI action of the form

$$
S_{\mathrm{DBI}}=-T_{D 7} \int \mathrm{d}^{8} \xi \operatorname{Str} \sqrt{\left|\operatorname{det}\left(P[g]+2 \pi \alpha^{\prime} F\right)\right|},
$$

where Str stands for the symmetrized trace, and $P[g]$ is the pull-back of the ten-dimensional metric to the worldvolume of the D7 branes. $F$ is the field strength tensor for the $U(2)$ gauge field on the 


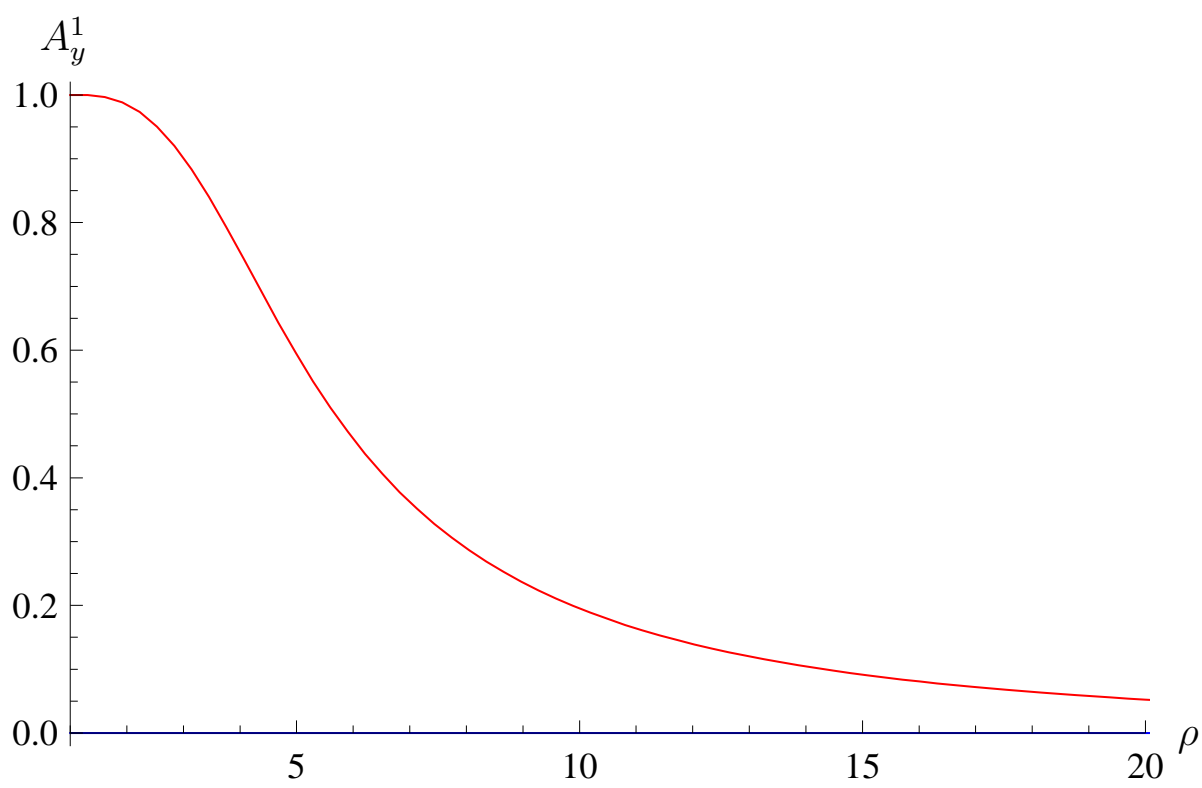

Figure 1: The profile of $A_{y}^{1}$ : This plot shows the profile of the gauge field $A_{y}^{1}$ dual to the condensate $J_{y}^{1}$ in the AdS radial direction. In agreement with spontaneous symmetry breaking, the field goes to zero at the boundary $\rho \rightarrow \infty$.

brane. We used two different prescriptions for evaluating the action and its equations of motion: a modified symmetrized trace prescription in analogy to [13], which allows for an all-order evaluation of the action, and the expansion of the DBI action to fourth order. Both approaches lead to qualitatively agreeing results.

The equations of motion obtained from (2.6) with (2.5) allow for a new solution with both non-zero $A_{t}^{3}$ and $A_{y}^{3}$, whose leading asymptotic form near the boundary is

$$
A_{t}^{3} \sim \mu-\frac{d_{t}^{3}}{\rho^{2}}, \quad A_{y}^{1} \sim-\frac{d_{y}^{1}}{\rho^{2}},
$$

where we have defined the dimensionless variable $\rho=r / r_{H}$. The full solution has to be obtained numerically. The profile for $A_{y}^{1}$ is shown in figure 1 .

$A_{t}^{3}$ contains the isospin chemical potential $\mu$ as well as the isospin density $d_{t}^{3}$. On the other hand, the source term in $A_{y}^{3}$ is absent, such that the leading term in $A_{y}^{3}$ corresponds to a VEV for the operator

$$
J_{y}^{1} \propto \bar{\psi} \sigma^{1} \gamma_{y} \psi+\phi \sigma^{1} \partial_{y} \phi=\bar{\psi}_{u} \gamma_{y} \psi_{d}+\bar{\psi}_{d} \gamma_{y} \psi_{u}+\text { bosons }
$$

and thus to spontaneous breaking of the symmetry $U(1)_{3}$. Simultaneously, the spatial rotational group $S O(3)$ is broken down to $S O(2)$. Using the method of holographic renormalisation [14] we calculated the on-shell action which determines the grand potential. Since the grand potential is smaller in the superfluid phase than in the normal phase, we see that the superfluid phase is thermodynamically preferred.

The non-trivial profile of the gauge field $A_{y}^{1}$ is induced by the non-Abelian interaction with the gauge field $A_{t}^{3}$ which generates an effective mass for the gauge field $A_{y}^{1}$. This mass generation 
is due to a Higgs mechanism since the $S U(2)$ symmetry is spontaneously broken in the bulk. The effective mass is given by $m^{2} \propto g^{t t} g^{y y}\left(A_{t}^{3}\right)^{2}$ which is negative in the bulk since $g^{t t}<0$. This triggers an instability such that the field $A_{y}^{1}$ condenses.

Moreover, by using the Kubo formula

$$
\sigma(\omega)=\frac{\mathrm{i}}{\omega} G^{R}(\omega, q=0),
$$

we may calculate the frequency-dependent conductivity $\sigma(\omega)$. $G^{R}$ is the retarded Green function of the current $J_{x}^{3}$ dual to a fluctuation of the gauge field component $a_{x}^{3}$, which we calculate using the method obtained in [15]. The current $J_{x}^{3}$ is the analogue of the electric current since it is charged under the $U(1)_{3}$ symmetry. In real space it is transverse to the condensate. Since this fluctuation is the only one which transforms as a vector under the $S O(2)$ rotational symmetry, it decouples from the other fluctuations of the system. The conductivity obtained by applying (2.9) to the retarded Green function for $J_{x}^{3}$ is displayed in figure 2. It shows the appearance and growth of a gap as we decrease the temperature, in agreement with expectations from superconductivity. Both calculational prescriptions - the adapted symmetrized trace prescription and the expansion of the DBI action to fourth order in the field strength - yield qualitatively very similar results. Using the Kramers-Kronig relation, which connects the real and imaginary part of the complex conductivity, we find a delta peak at $\omega=0$ in the real part of the conductivity, $\operatorname{Re} \sigma(\omega) \propto \pi n_{s} \delta(\omega)$ in the condensed phase. As expected from Ginzburg-Landau theory, our numerics show that the superconducting density $n_{s}$ vanishes linearly at the critical temperature, $n_{s} \propto\left(1-T_{c} / T\right)$ for $T \approx T_{c}$. Note that for translation invariant systems at finite density, there is a delta peak in the real part of the conductivity even in a normal conducting phase since the charge carriers cannot lose their momentum. This peak is called Drude peak. In our system, however, the charge carriers can dissipate their momentum although our system is translation invariant. The adjoint degrees of freedom can transfer momentum at order $N_{c}^{2}$ while the fundamental degrees of freedom only at order $N_{c}$. The adjoint degrees of freedom effectively act as a heat sink into which the flavor fields can dissipate their momentum. Thus we do not observe a Drude peak in our system.

Let us now compare our results to QCD. In QCD, the pion condensate is of course the natural state in isospin asymmetric matter. The condensation of a particle sets in if the isospin chemical potential is larger than the mass of this particle. According to this rule, the pions condense first in QCD since they are the Nambu-Goldstone bosons of the spontaneous chiral symmetry breaking and therefore the lightest particles. However the dual field theory which we consider here is supersymmetric at zero temperature and therefore chiral symmetry cannot be broken spontaneously. In this supersymmetric theory, the vector and scalar mesons have the same mass at zero temperature. Due to finite temperature effects, the mass of the vector and scalar mesons can become different as we increase the temperature. It is a priori unclear which particle will condense. In our model we checked that the vector mesons condense first such that the $\rho$ meson condensation state, which we describe here, is the physical ground state of our system near the phase transition.

\section{Holographic superfluid generated by an external B field}

Let us now turn to the possibility of a transition to a superfluid phase with condensed $\rho$ mesons which is exclusively induced by a finite magnetic field $B$. In QCD this effect was found recently in 


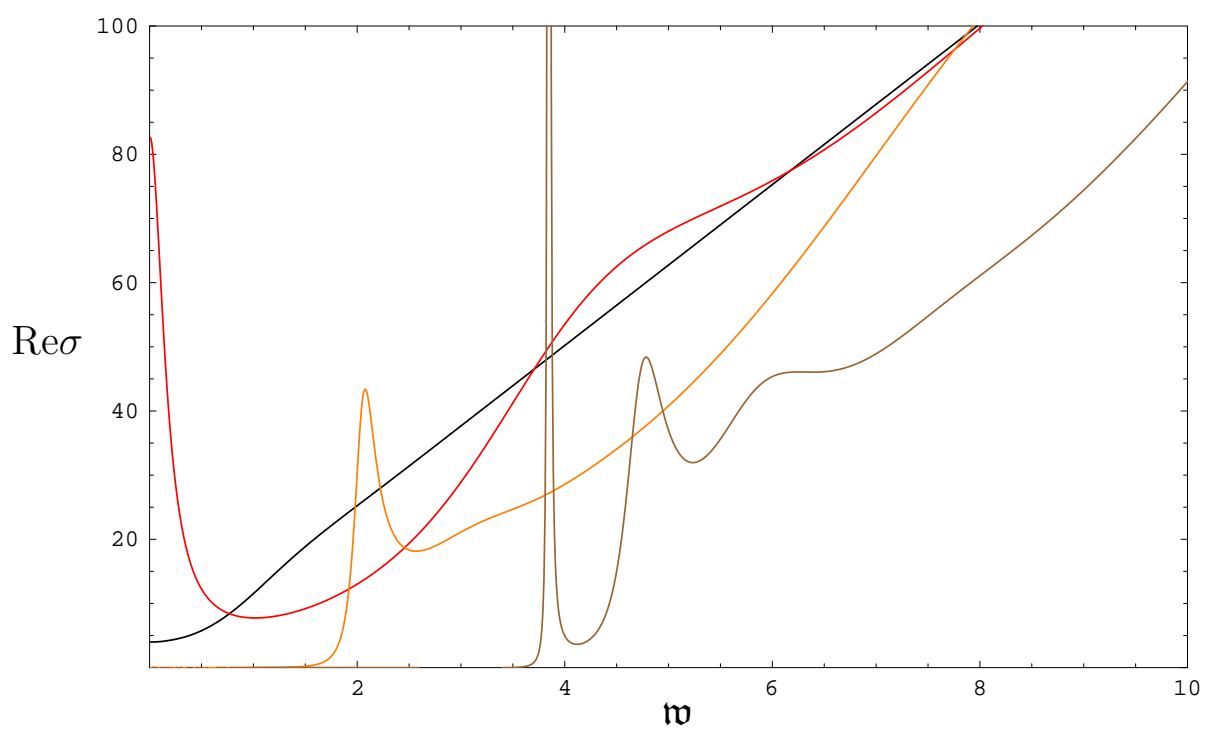

Figure 2: Real part of conductivity, Re $\sigma$, in units of $N_{f} N_{c} T /(16 \pi)$ versus the dimensionless frequency $\mathfrak{w}=\omega /(2 \pi T)$ for massless quarks computed from the adapted symmetrized trace prescription for the isospin background. Distinct curves correspond to $T / T_{c}=\infty$ (black), 1(red), 0.5 (orange) and 0.28 (brown). By decreasing the temperature below the critical one, a gap appears which is a characteristic feature of a superconductor. In addition prominent peaks arise, corresponding to mesonic excitations. Figure from [5].

$[10,11,12]$. In order to simplify the discussion, we consider the DBI action (2.6) to lowest order only, i.e. to $\mathscr{O}\left(\alpha^{\prime 2}\right)$. In addition we assume that the fields do not depend on the coordinates of the $S^{3}$, such that we can integrate out this directions. In this limit the effective action for the gauge field on the branes is the Yang-Mills action

$$
S=\frac{1}{4 \hat{g}^{2}} \int \mathrm{d}^{5} x \sqrt{-g} F_{\mu v}^{a} F^{a \mu v},
$$

where the Yang-Mills coupling $\hat{g}$ is determined by the brane tension and the volume of the $S^{3}$. In order to switch on a finite magnetic field $B$ in $z$ and flavor $\tau^{3}$ direction on the boundary field theory, we consider the non-zero field strength $F_{x y}^{3}$ in the bulk. Choosing the $B$ field in these directions breaks the spatial rotations $S O(3)$ down to $S O(2)$ and $S U(2)$ to $U(1)_{3}$. As before we now study the spontaneous breaking of the $U(1)_{3}$ symmetry which induces superfluidity.

By using the gauge symmetry, we may choose $A_{y}^{3}(x, r)$ to be the only non-zero gauge field component which introduces an $r$-dependent $B$ field. As seen in the section above, the condensation of the $\rho$ mesons is induced by the non-Abelian interaction with the background field, $A_{y}^{3}$ in this case. This suggests that we consider the gauge fields $A^{1,2}$ dual to the charged $\rho$ mesons which we expect to condense, and more precisely an ansatz with non-zero gauge fields $A_{x, y}^{1,2}$ in the $x$ or $y$ direction ${ }^{1}$. The equations of motion from the action (3.1) are

$$
\nabla_{\mu} F^{a \mu v}=-\varepsilon^{a b c} A_{\mu}^{b} F^{c \mu v},
$$

\footnotetext{
${ }^{1}$ Note that it is also consistent to consider an non-zero $A_{z}^{1,2}$ or $A_{t}^{1,2}$ exclusively. However this does not lead to a condensation.
} 
In the normal phase, i. e. when $A^{1,2} \equiv 0$, we have that $A_{y}^{3}=x B$ and the $B$ field is constant. Since we expect a second order phase transition to the superfluid phase, the condensate $\left\langle J_{x, y}^{1,2}\right\rangle$ is small slightly above the critical magnetic field $B_{c}$. Thus near the expected critical point, we may expand the solution of (3.1) in $\varepsilon \propto\left\langle J_{x, y}^{1,2}\right\rangle$, as done in [16, 17], giving

$$
\begin{aligned}
A_{y}^{3}(x, r) & =x B_{c}+\mathscr{O}\left(\varepsilon^{2}\right), \\
A_{x, y}^{1,2}(x, r) & =\varepsilon a_{x, y}^{1,2}(x, r)+\mathscr{O}\left(\varepsilon^{3}\right) .
\end{aligned}
$$

To first order in $\varepsilon$, finding the normalizable mode for $a_{x, y}^{1,2}$ is equivalent to finding the quasinormal modes with zero frequency in the normal phase. In order to find this quasinormal mode it is useful to consider gauge invariant fields $E_{x, y}^{ \pm}=A_{x, y}^{1} \pm \mathrm{i} A_{x, y}^{2}$, which allow us to reduce the equations of motion. Due to the non-Abelian interaction $\varepsilon^{a b c} A_{x}^{b} F^{c x y} \propto B\left(A_{x}^{2} \delta^{a 1}-A_{x}^{1} \delta^{a 2}\right.$ ) (and similarly for $A_{y}^{1,2}$ ) we expect a Zeeman splitting in the mass spectrum, such that one mode of $E^{ \pm}$gets a higher mass while the other mass gets lowered. We expect that by increasing the magnetic field we may get a negative mass state which will lead to meson condensation. For a more precise analysis, we plan to work out the meson spectrum in the normal phase to obtain the instability, and then to study the condensate which stabilizes the system.

For the Sakai-Sugimoto model of holographic QCD [18], which involves D8 and anti-D8 brane probes in a compactified D4 brane background, a related analysis will soon appear in [19] (see also the contribution [20] to these proceedings).

\section{Discussion}

We have reviewed recent results on a holographic superfluid in presence of an isospin chemical potential and density for which the dual field theory is explicitly known. Also, we have argued that by a related mechanism, a superfluid condensate corresponding to the charged $\rho$ mesons may arise also in presence of a magnetic field at vanishing isospin chemical potential and density. Let us add a few comments about this result:

- In view of our setup using flavour D7 branes, we have a flavour superfluid and not an electromagnetic superconductor. The spontaneously broken $U(1)_{3}$ is global in the dual field theory. Also, our magnetic field is a flavour magnetic field rather than the standard magnetic field of electromagnetism. In the QCD approach [10, 11, 12], the condensation mechanism arises from a non-minimal coupling of the charged $\rho$ mesons to the electromagnetic field. We do not have such an interaction here.

- In the simple case considered here, our superfluid is anisotropic as in $[10,11,12]-$ the $\rho$ meson condensate will point in the direction perpendicular to the B field. Moreover, we expect our condensate to be dependent on the direction transverse to the magnetic field as observed in $[10,11,12]$. 
- For simplicity we have considered a massless flat D7 brane embedding. For a complete analysis, it will also be important to take into account the chiral symmetry breaking induced by the B field, known as magnetic catalysis, which leads to a bending of the $\mathrm{D} 7$ brane probes, as observed for instance in [21, 22, 23].

We plan to explore these issues in more detail in the future.

\section{Acknowledgements}

We are grateful to our collaborators M. Ammon, M. Kaminski and A. O'Bannon. J.E. would like to thank the organisers of the Workshop 'The many faces of QCD' in Gent for a pleasant and stimulating conference, as well as M. Chernodub and H. Verschelde for discussions.

\section{References}

[1] S. S. Gubser, "Breaking an Abelian gauge symmetry near a black hole horizon," Phys. Rev. D78 (2008) 065034. [arXiv:0801.2977 [hep-th]].

[2] S. A. Hartnoll, C. P. Herzog, G. T. Horowitz, "Building a Holographic Superconductor," Phys. Rev. Lett. 101 (2008) 031601. [arXiv:0803.3295 [hep-th]].

[3] S. S. Gubser, S. S. Pufu, "The Gravity dual of a p-wave superconductor," JHEP 0811 (2008) 033. [arXiv:0805.2960 [hep-th]].

[4] M. Ammon, J. Erdmenger, M. Kaminski and P. Kerner,, "Superconductivity from gauge/gravity duality with flavor,” Phys. Lett. B680 (2009) 516-520. [arXiv:0810.2316 [hep-th]].

[5] M. Ammon, J. Erdmenger, M. Kaminski and P. Kerner, "Flavor Superconductivity from Gauge/Gravity Duality,” JHEP 0910 (2009) 067. [arXiv:0903.1864 [hep-th]].

[6] J. P. Gauntlett, J. Sonner, T. Wiseman, "Holographic superconductivity in M-Theory," Phys. Rev. Lett. 103 (2009) 151601. [arXiv:0907.3796 [hep-th]].

[7] S. S. Gubser, S. S. Pufu, F. D. Rocha, "Quantum critical superconductors in string theory and M-theory,” Phys. Lett. B683 (2010) 201-204. [arXiv:0908.0011 [hep-th]].

[8] O. Aharony, K. Peeters, J. Sonnenschein et al., "Rho meson condensation at finite isospin chemical potential in a holographic model for QCD," JHEP 0802 (2008) 071. [arXiv:0709.3948 [hep-th]].

[9] M. Ammon, J. Erdmenger, M. Kaminski and A. O’Bannon, "Fermionic Operator Mixing in Holographic p-wave Superfluids,” JHEP 1005 (2010) 053. [arXiv:1003.1134 [hep-th]].

[10] M. N. Chernodub, "Superconductivity of QCD vacuum in strong magnetic field," Phys. Rev. D82 (2010) 085011. [arXiv:1008.1055 [hep-ph]].

[11] M. N. Chernodub, "Electromagnetically superconducting phase of QCD vacuum induced by strong magnetic field,"

[arXiv:1011.2658 [hep-ph]].

[12] M. N. Chernodub, "Spontaneous electromagnetic superconductivity of vacuum in strong magnetic field: an evidence from the Nambu-Jona-Lasinio model," [arXiv:1101.0117 [hep-ph]].

[13] N. R. Constable, R. C. Myers, O. Tafjord, “The Noncommutative bion core,” Phys. Rev. D61 (2000) 106009. [hep-th/9911136]. 
[14] K. Skenderis, "Lecture notes on holographic renormalization,” Class. Quant. Grav. 19 (2002) 5849-5876. [hep-th/0209067].

[15] D. T. Son, A. O. Starinets, "Minkowski space correlators in AdS / CFT correspondence: Recipe and applications,” JHEP 0209 (2002) 042. [hep-th/0205051].

[16] C. P. Herzog, S. S. Pufu, “The Second Sound of SU(2),” JHEP 0904, 126 (2009). [arXiv:0902.0409 [hep-th]].

[17] C. P. Herzog, “An Analytic Holographic Superconductor,” Phys. Rev. D81, 126009 (2010). [arXiv:1003.3278 [hep-th]].

[18] T. Sakai, S. Sugimoto, “Low energy hadron physics in holographic QCD,” Prog. Theor. Phys. 113 (2005) 843-882. [hep-th/0412141].

[19] N. Callebaut, D. Dudal and H. Verschelde, to appear.

[20] N. Callebaut, D. Dudal, H. Verschelde, "Holographic study of rho meson mass in an external magnetic field", in these proceedings.

[21] V. G. Filev, C. V. Johnson, R. C. Rashkov and K.S. Viswanathan, "Flavoured large N gauge theory in an external magnetic field,” JHEP 0710 (2007) 019. [hep-th/0701001].

[22] J. Erdmenger, R. Meyer, J. P. Shock, "AdS/CFT with flavour in electric and magnetic Kalb-Ramond fields,” JHEP 0712 (2007) 091. [arXiv:0709.1551 [hep-th]].

[23] T. Albash, V. G. Filev, C. V. Johnson and A. Kundu, "Finite temperature large N gauge theory with quarks in an external magnetic field," JHEP 0807 (2008) 080. [arXiv:0709.1547 [hep-th]]. 